\title{
Análisis de la Evaluación del Programa Nacional de Posgrados de Calidad: Universidad de Sonora 2015-2018
}

\author{
Romero Borbón Luis Felipe ${ }^{1}$, Borbón Morales Carlos Gabriel ${ }^{1}$, Romero Dessens Luis Felipe², \\ Rueda Puente Edgar Omar², Ortega García Jesús ${ }^{3}$ \\ 1 Centro de Investigación en Alimentación y Desarrollo, Hermosillo, Sonora, México. \\ 2 Universidad de Sonora, Hermosillo, Sonora, México. \\ 3 Universidad de Sonora, Caborca, Sonora, México.
}

\section{Resumen}

La gestión de la calidad en los programas de posgrado en México, de las Instituciones de Educación Superior (IES) y Centros Públicos de Investigación $(\mathrm{CPI})$, se considera que aún está en proceso de evolución; ya que hace relativamente poco tiempo que la difusión de los resultados de la calidad de dichos programas académicos es de acceso público. Presentados a manera de encuestas y en forma de datos abiertos, si bien cumplen con las normas de transparencia dictadas por entidades gubernamentales. Esta información está referida a manera de una encuesta, que incluye respuestas categóricas y de opinión, establecidas en los dictámenes de evaluación del Programa Nacional de Posgrados de Calidad (PNPC). Con base en dicha información, esta investigación busca Análisis de Resultados de Dictámenes de Evaluación del Programa Nacional de Posgrados de Calidad, tomando como ejemplo los programas académicos de la Universidad de Sonora.

Palabras Clave: PNPC, Gestión de la Calidad, IES.

\section{Analysis of the Evaluation of the National Quality Postgraduate Program: Universidad de Sonora 2015-2018}

\begin{abstract}
Quality management of mexican Universities and Public Research Centers, is considered to be still in the process of evolution; since results of the quality of the academic programs have been publicly available for a relatively short time. Presented in the form of surveys and in the form of open data, although they comply with the transparency standards established by government entities. This information is referred to as a survey, which includes categorical and opinion responses, referred to in the assessment opinions of the National Quality Postgraduate Program in México. This research seeks Analysis of Results of Assessment Opinions of the National Quality Postgraduate Program, using as example the academic programs of the University of Sonora
\end{abstract}

Keywords: PNPC, quality managment. Universities. 


\section{INTRODUCCION}

El capital humano y el conocimiento en un país tienden a ser un impacto positivo sobre la productividad. Entendiendo por capital humano a la mezcla de aptitudes y habilidades innatas a las personas, así como la calificación y el aprendizaje que adquieren en la educación y la capacitación (OCDE, 2007).

A juicio de la OCDE (2011), las regiones que están impulsando el uso de capital humano como motor del dinamismo económico se están convirtiendo también en regiones innovadoras.

De ahí que un criterio de mejora para la formación del capital humano, es la evaluación que las las Instituciones de Educación Superior (IES) realizan a sus programas educativos, estos han sido implementados desde fines de los años 1960 para estudiar y reformar las universidades. Donde los criterios de evaluación suelen responder a exigencias del control de gestión, de la evaluación de resultados o de procesos internos. Donde el análisis de indicadores, está normalmente vinculado a requisitos de carácter institucional (Fernández, 2007).

En México, la instancia encargada de evaluar a los programas de posgrado de las IES y los CPI, es el Consejo Nacional de Ciencia y Tecnología (CONACYT); mediante el Programa Nacional de Posgrados de Calidad (PNPC), quien ha definido un conjunto de políticas, lineamientos y criterios, establecidos en su modelo de evaluación. De ahí la importancia para una IES, de contar con un posgrado dentro del PNPC; ya que es un referente confiable de la calidad de la oferta educativa y la formación de recursos humanos de alto nivel (CONACYT, 2019).

Como indica el Marco de Referencia del PNPC (2020), el modelo contiene cinco categorías conformadas por diferentes criterios, bajo el paradigma de: formacióninvestigación-innovación-vinculación, buscan evaluar a los programas. Estas categorías son: Contexto y responsabilidad social, estructura e infraestructura del programa, procesos académicos, pertinencia del programa, y resultados e impacto. Los programas que cumplan con los dichos criterios y aprueben el proceso de evaluación, son integrados al PNPC, y según su nivel de cumplimiento, obtienen el nivel correspondiente a las características del programa; competencia internacional, consolidado, en desarrollo y de reciente creación.

\section{1.- El PNPC en México}

Según el padrón del PNPC (2020), en México existen 2,382 programas de posgrado reconocidos por el PNPC. De los cuales 1,522 son programas enfocados a la investigación y 857 enfocados al ámbito profesional. A su vez, distribuidos en siete categorías según el área de conocimiento: físicomatemáticas y ciencias de la tierra, biología y química, medicina y ciencias de la salud, humanidades y ciencias de la conducta, ciencias sociales, biotecnología y ciencias agropecuarias, e ingenierías.

Más de la mitad de los programas de posgrado corresponden al de nivel maestría, poco menos de la tercera son doctorados, y menos de una sexta parte son de especialidad, ver figura 1.

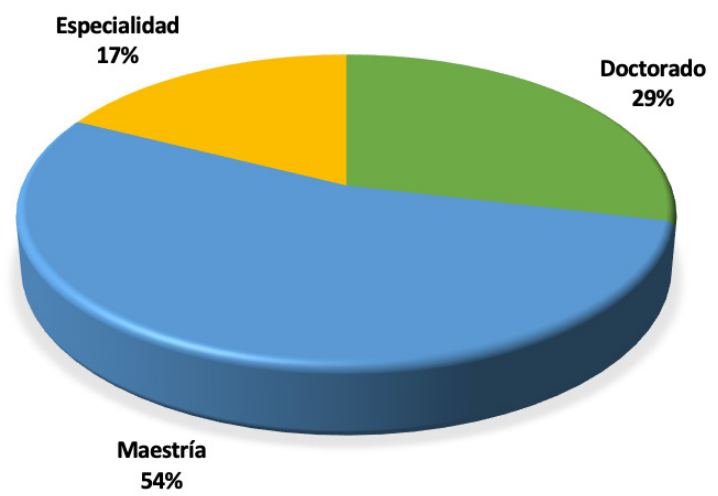

Figura1. México: Programa de Posgrado Acreditados por PNPC, 2019. Fuente Elaboración propia con base en: Datos Abiertos del PNPC. México. http://svrtmp.main.conacyt.mx/ ConsultasPNPC/datos-abiertos-pnpc.php

En relación al nivel de consolidación de los programas de posgrado, se observa en la gráfica 2, destaca la participación de las maestrías con más de $50 \%$. Seguido por los programas de doctorado con $23 \%$. Por último, las especialidades entre $10 \%$ y hasta el $23 \%$.

\section{2.- El PNPC en Sonora}

En el Estado de Sonora, se cuenta con 57 programas PNPC, en las siguientes instituciones: Universidad de Sonora (UNISON) 68\%; Instituto Tecnológico de Sonora (ITSON) 14\%; Centro de Investigación en Alimentación y Desarrollo (CIAD) 7\%; Instituto Tecnológico de Hermosillo (ITH) 5\%; El Colegio de Sonora (COLSON) 4\%; Universidad Estatal de Sonora (UES) 2\%; e Instituto Tecnológico de Cajeme (ITESCA) $2 \%$. En la tabla 1, se muestra la cantidad de programas de cada institución según el nivel obtenido (CONACYT, 2020b).

\section{El concepto de calidad en los posgrados PNPC.}

La definición de calidad, es la establecida por la Organización 
Tabla 1. Programas de posgrado por institución y nivel alcanzado en su evaluación del PNPC.

\begin{tabular}{lccccc} 
Institución & $\begin{array}{c}\text { Nivel de PNPC obtenido } \\
\text { Reciente } \\
\text { creación }\end{array}$ & $\begin{array}{c}\text { En } \\
\text { desarrollo }\end{array}$ & Consolidado & $\begin{array}{c}\text { Competencia } \\
\text { internacional }\end{array}$ & Total \\
\hline UNISON & 10 & 7 & 19 & 2 & 38 \\
\hline ITSON & 2 & 4 & 2 & - & 8 \\
\hline CIAD & - & 1 & 1 & 2 & 4 \\
\hline ITH & 1 & 2 & - & - & 3 \\
\hline COLSON & - & - & 1 & 1 & 2 \\
\hline UES & - & 1 & - & - & 1 \\
\hline ITESCA & - & 1 & - & - & 1 \\
\hline Total & 13 & 16 & 23 & 5 & 57 \\
\hline
\end{tabular}

Fuente: Elaboración propia con base en el Padrón de posgrados del PNPC. 2019

Internacional de Normalización (ISO, 2015). Que en su norma ISO 9000: 2015, la precisa como: el grado en el que un conjunto de características inherentes de un objeto, cumple con los requisitos de: necesidad o expectativa establecida, generalmente explícita u obligatoria, esta conceptualización es la base de la calidad de toda empresa, sin importar el rubro al que se dedique.

En particular, para la definición de calidad de los posgrados, autores como, Careaga (2017), Fatemeh (2011) y Taraneh (2012), coinciden con lo establecido por la UNESCO (2009), la cual establece que se deben incluir todas las funciones y actividades involucradas en el proceso educativo: la enseñanza, investigación, personal, estudiantes, instalaciones y equipos, entre otros.

Por su parte, el PNPC, define calidad, como: "Grado en el que un conjunto de rasgos diferenciadores inherentes a los programas educativos cumple con una necesidad o expectativa establecida. Propiedad de un programa de posgrado que cumple los criterios o estándares previamente establecidos en el marco de referencia". (PNPC, 2011, p,6).

En este sentido, el aseguramiento de la calidad se convierte en una de las principales preocupaciones de las IES y CPI en México, ya que su propósito no sólo es impartir una educación de excelencia y sobresalir ante su competencia, sino que también es brindar beneficios para el estudiante como: Reconocimiento por la SEP y el CONACYT, becas a estudiantes de tiempo completo, y recursos para los proyectos de investigación del programa.

Asimismo, la tarea educativa también tiene el sentido de la responsabilidad social, tal como lo menciona Pérez (2007), para ser más productivas, en su relación con su entorno, las instituciones deben estar dispuestas a conocerse mejor y a evaluar el cumplimiento de sus responsabilidades de cara a la sociedad.

\section{Gestión del conocimiento}

Tarí y García (2009) establecen que la gestión del conocimiento (GCo), es el proceso dinámico de: creación, almacenamiento, transferencia-aplicación, y uso del conocimiento, con el fin de mejorar los resultados en una organización. Así, la GCo está íntimamente relacionada con la gestión de calidad en la educación.

Para las IES y los CPI, la GCo es primordial, en especial el conocimiento científico; pues su visión y misión implica armonizar la creación, adquisición, preservación y transferencia del conocimiento en un ambiente de responsabilidad social y de cooperación al desarrollo integral local y nacional (Barroso, 2011).

En este orden de ideas, la importancia del uso de la GCo en ámbitos de las organizaciones e instituciones públicas, como componente para la consecución de los objetivos institucionales. Ha propiciado que instancias gubernamentales como la Secretaría de Función Pública (2018), hayan expedido los Marcos Conceptuales sobre la GCo, y así contribuir al entendimiento de la competitividad de la empresa y la eficiencia del sector público.

Barbón y Fernández (2017) comentan que la integración de la GCo, la ciencia, la tecnología y la innovación, son elementos promotores del establecimiento de estrategias, 
para reducir y eliminar brechas en factores problemáticos para los posgrados.

El objetivo del presente estudio es: analizar los dictámenes de los programas de posgrado de la Universidad de Sonora, dentro del marco de la evaluación del PNPC, para identificar factores de mejora en la operación, las políticas, los lineamientos, y los criterios, en el período 2015-2019.

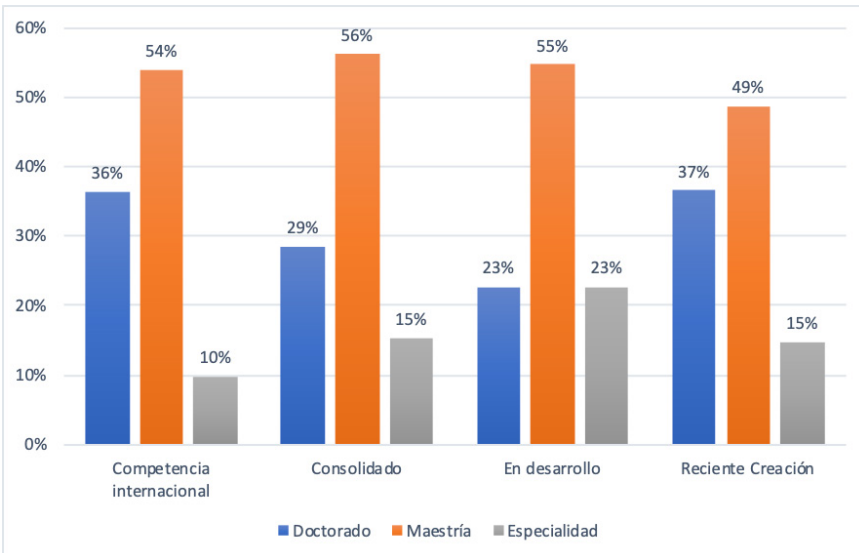

Fïigura 2. México: Programas de Posgrado de acuerdo a su nivel de consolidación, 2019. Fuente: Elaboración propia con base en: Datos Abiertos del PNPC. México. http:// svrtmp.main.conacyt. $\mathrm{mx} /$ ConsultasPNPC/datos-abiertospnpc.php

\section{MATERIALES Y MÉTODOS}

Para fines de este análisis, se utilizaron los dictámenes de evaluación para los posgrados de la Universidad de Sonora, que actualmente tienen reconocimiento PNPC y están orientados a la investigación. Lo anterior con base en dos aspectos: la primera es que los posgrados en investigación son más numerosos que aquellos profesionalizantes, y segundo, para minimizar las diferencias de los documentos, requisitos y criterios de evaluación entre los programas, ya que según su enfoque existe disparidad entre ellos.

Se utilizó una muestra de 28 programas, con sus respectivos dictámenes, los cuales se obtuvieron del portal de la Dirección de Investigación y Posgrado (2020) de la Universidad de Sonora. En la tabla 2, se clasifican según la división a la que pertenecen: Ciencias Biológicas y de la Salud, Ciencias Exactas y Naturales, Ciencias Sociales, Humanidades y Bellas Artes, Ingeniería y Ciencias Económicas y Administración.

Se identificaron un total de 127 criterios: Compromiso institucional, estructura y personal académico del programa, estudiantes, infraestructura del programa, resultados y vinculación, y plan de mejora. Como parte de la evaluación, al final de cada dictamen se hace una reflexión por parte del comité de pares ciegos quienes realizaron la evaluación,
El análisis se realizó en dos etapas, la primera consistió en especificar el desempeño de los programas con respecto al cumplimiento de los criterios de evaluación. En la segunda etapa, está enfocada en los criterios de reflexión donde se capta la apreciación de los evaluadores pares. Finalmente, la discusión de los resultados se realizará a través de un análisis FODA donde se resaltarán las Fortalezas, Oportunidades, Debilidades y Amenazas encontradas en el análisis.

\section{RESULTADOS Y DISCUSION}

Los resultados obtenidos del análisis realizado a los dictámenes de evaluación de los programas de posgrado se presentarán según la etapa a la que pertenecen de la siguiente manera: La primer etapa, desempeño de los programas, los cuales se agruparon por la división a la que pertenecen dentro del organigrama de la Universidad de Sonora, e incluye la siguiente información: Número y porcentaje de criterios que fueron relevantes dentro del dictamen (estos son aquellos que fueron evaluados o estaban considerados dentro del reporte), el porcentaje de cumplimiento de los criterios según la categoría a la que pertenecen y finalmente se destacan aquellos criterios que fueron observados recurrentemente dentro de las evaluaciones para los programas de la división.

La segunda etapa, criterios de reflexión, son aquellos que fueron recurrentes para todos los programas, se muestra el número y porcentaje de los programas que cumplen con el indicador según la apreciación de los evaluadores pares, y este considera el total de los programas evaluados.

\section{División de Ciencias Biológicas y de la Salud}

La integran seis programas PNPC, tres en nivel consolidado y otros tres en desarrollo. De los 127 criterios generales, 61 fueron considerados relevantes, $66 \%$ han sido cumplidos por todos los programas, y $34 \%$ están en proceso de cumplimiento. Los resultados de cumplimiento de estos indicadores por categoría fueron: Compromiso institucional 90\%; estructura y personal académico 96.6\%; estudiantes 87.8\%; infraestructura del programa $100 \%$; resultados y vinculación $84.1 \%$; y plan de mejora - 83.3\%.

Los criterios en proceso de cumplimiento, se relacionan con la comunicación constante con sus egresados y la organización de actividades académicas, y el segundo con los resultados y beneficios de las acciones de vinculación en los sectores sociales.

\section{División de Ciencias Exactas y Naturales}

En esta división hay ocho programas PNPC, uno de ellos de competencia internacional, seis consolidados y uno 
Tabla 2. Programas de la Universidad de Sonora con reconocimiento a su calidad por el PNPC.

\begin{tabular}{|c|c|c|}
\hline Dependencia & Programa & Nivel de PNPC \\
\hline \multirow{6}{*}{$\begin{array}{l}\text { División de Ciencias } \\
\text { Biológicas y de la Salud }\end{array}$} & Doctorado en Biociencias & En desarrollo \\
\hline & Posgrado en Biociencias & En desarrollo \\
\hline & Doctorado en Ciencias de los Alimentos & Consolidado \\
\hline & Maestría en Ciencia y Tecnología de Alimentos & Consolidado \\
\hline & Doctorado en Ciencias Químico Biológicas y de la Salud & En desarrollo \\
\hline & Maestría en Ciencias de la Salud & Consolidado \\
\hline \multirow{8}{*}{$\begin{array}{l}\text { División de Ciencias } \\
\text { Exactas y Naturales }\end{array}$} & Doctorado en Ciencias (Física) & Consolidado \\
\hline & Maestría en Ciencias: Física & Competencia Internacional \\
\hline & Doctorado en Ciencias Matemáticas & Consolidado \\
\hline & Maestría en Ciencias Matemáticas & Consolidado \\
\hline & Maestría en Ciencias - Geología & Consolidado \\
\hline & Doctorado en Nanotecnología & Consolidado \\
\hline & Maestría en Nanotecnología & Consolidado \\
\hline & Maestría en Ciencias en Electrónica & Reciente creación \\
\hline \multirow{6}{*}{$\begin{array}{l}\text { División de Ciencias } \\
\text { Sociales }\end{array}$} & Doctorado en Innovación Educativa & Reciente creación \\
\hline & Maestría en Innovación Educativa & Consolidado \\
\hline & Posgrado Integral en Ciencias Sociales & Consolidado \\
\hline & Maestría en Ciencias Sociales & Consolidado \\
\hline & Doctorado en Psicología & Reciente creación \\
\hline & Maestría en Psicología & Reciente creación \\
\hline \multirow{3}{*}{$\begin{array}{l}\text { División de } \\
\text { Humanidades y } \\
\text { Bellas Artes }\end{array}$} & Doctorado en Humanidades & En desarrollo \\
\hline & Maestría en Lingüística & Consolidado \\
\hline & Maestría en Literatura Hispanoamericana & Consolidado \\
\hline \multirow{4}{*}{ División de Ingeniería } & Doctorado en Ciencia de Materiales & En desarrollo \\
\hline & Maestría en Ciencias de Materiales & Consolidado \\
\hline & Doctorado en Ciencias de la Ingeniería: Ingeniería Química & En desarrollo \\
\hline & Maestría en Ciencias de la Ingeniería: Ingeniería Química & Consolidado \\
\hline $\begin{array}{l}\text { División de Ciencias } \\
\text { Económicas y } \\
\text { administración }\end{array}$ & Maestría en Integración Económica & En desarrollo \\
\hline
\end{tabular}

Fuente: Elaboración propia con base en datos de la Universidad de Sonora.

de reciente creación. Del total de los criterios, 64 fueron relevantes, $56 \%$ han sido cumplidos en su totalidad por todos los programas, y $44 \%$ están en proceso de cumplimiento. Los resultados de cumplimiento de estos indicadores por categoría fueron: Compromiso institucional 98.6\%; estructura y personal académico $94.4 \%$; estudiantes $93.1 \%$; infraestructura del programa $100 \%$; resultados y vinculación $89.58 \%$; y plan de mejora $85 \%$.

En relación a los criterios que están en proceso de cumplimiento está el relacionado con la comunicación constante de egresados y la organización de actividades académicas con ellos.

\section{División de Ciencias Sociales}

Cuenta con seis programas PNPC, tres consolidados y tres de reciente creación. Del total de los criterios, 55 fueron considerados relevantes, $85 \%$ han sido cumplidos por todos los programas, y $15 \%$ en proceso de cumplimiento. Los resultados de cumplimiento de estos indicadores por categoría fueron: Compromiso institucional 100\%; estructura y personal académico 98.3\%; estudiantes 100\%; infraestructura del programa $100 \%$; resultados y vinculación $92.42 \%$; y plan de mejora $100 \%$. 


\section{División de Humanidades y Bellas Artes}

Esta división tiene tres programas PNPC, dos consolidados y uno en desarrollo. Del total de los criterios, 70 de los indicadores fueron considerados relevantes, $80 \%$ han sido cumplidos por todos los programas, y $20 \%$ en proceso de cumplimiento. Los resultados de cumplimiento de estos indicadores por categoría fueron: Compromiso institucional 100\%; estructura y personal académico $90 \%$; estudiantes 97.6\%; infraestructura del programa $100 \%$; resultados y vinculación $82.4 \%$; y plan de mejora $86.7 \%$.

Existen tres criterios que están en proceso de cumplimiento, el primero de ellos relacionado con la comunicación constante con sus egresados y la organización de actividades académicas con ellos, el segundo corresponde a la comunicación constante y de colaboración con los sectores de la sociedad, y finalmente referente si el plan de mejora y sus acciones, permiten ver la evolución positiva del programa y su tránsito natural al nivel superior.

\section{División de Ingeniería}

Cuenta con cuatro programas PNPC, dos consolidados y otros dos en desarrollo. Del total de criterios, 69 fueron considerados relevantes, $55 \%$ han sido cumplidos en su totalidad por todos los programas, y $45 \%$ en proceso de cumplimiento. Los resultados de cumplimiento de estos indicadores por categoría fueron: Compromiso institucional 95\%; estructura y personal académico $83.3 \%$; estudiantes 84.6\%; infraestructura del programa $100 \%$; resultados y vinculación $60.5 \%$; y plan de mejora $62.5 \%$.

Cuatro son los criterios que están en proceso de cumplimiento: seguimiento adecuado de estrategias y acciones en el plan de mejora; efectividad de la participación en los estudiantes en la productividad del programa; comunicación constante con sus egresados y la organización de actividades académicas; y el último criterio en proceso de cumplimiento concurrente en los programas de ingeniería es la actualización y revisión periódica del plan de estudios.

\section{División de Ciencias Económicas y administración}

EN esta división, hay un programa PNPC, mismo que actualmente está en desarrollo. La relación de relevancia de los criterios relevantes para estos programas, 70 de los indicadores fueron considerados relevantes, $100 \%$ han sido cumplidos en su totalidad por el programa.

\section{Criterios de reflexión}

A continuación, se muestran los resultados obtenidos a los principales criterios de reflexión:

1.- La evolución del programa tiene tendencia positiva en la pertinencia para el ámbito regional, nacional o internacional. Lo cumplen 17 programas (61\%).

2.- Existen estructuras y experiencia para orientar al posgrado a la realización de proyectos que atienden las necesidades del programa o demandas de los sectores de la sociedad. Estos son 22 programas que lo cumplen (79\%).

3.- Las líneas de generación y aplicación del conocimiento, son adecuadas para generar proyectos y tesis que aborden problemas complejos con enfoque multidisciplinario. Lo cumplen 20 programas (71\%).

4.- El posgrado se puede calificar como innovador", 13 lo cumplen $(46 \%)$

\section{Discusión de los Resultados}

Usando como base la tabla 4, se observa que la infraestructura de los programas de la institución es la principal fortaleza, ya que en todos los programas el cumplimiento de los criterios fue del $100 \%$, seguido de compromiso institucional con un $97 \%$, estructura y personal académico del programa y estudiantes con un $94 \%$. En las categorías de resultados y vinculación, así como plan de mejora es donde se encuentra los resultados más bajos en cumplimiento de los criterios de las categorías con un 85 y $86 \%$ respectivamente.

De acuerdo al análisis FODA de lo observado durante la investigación, se citarán los puntos más relevantes encontrados:

Fortalezas: Los núcleos académicos básicos dentro de los programas; los planes de estudio en su mayoría son pertinentes, actualizados y relevantes para el entorno; las líneas de generación y/o aplicación del conocimiento son congruentes con el perfil de egreso, el perfil de los profesores, la productividad académica y la orientación del programa.

Debilidades: El seguimiento de egresados; matrícula activa baja: baja vinculación de los programas con el sector productivo.

Oportunidades: infraestructura y compromiso institucional; la Institución con el mayor número de programas reconocidos por su calidad en el Estado; la experiencia y conocimiento de los programas de competencia internacional y consolidados. 
Tabla 3. Análisis FODA de los resultados obtenidos en la investigación.

\begin{tabular}{|c|c|}
\hline Fortalezas & Oportunidades \\
\hline $\begin{array}{l}\text { Núcleos académicos básicos cumplen gran parte } \\
\text { de los indicadores (SNI, perfil deseable, publica- } \\
\text { ciones). } \\
\text { Los planes de estudio son pertinentes y relevantes } \\
\text { para el entorno. } \\
\text { Las líneas de generación y/o aplicación del cono- } \\
\text { cimiento son congruentes con el perfil de egreso, } \\
\text { el perfil de los profesores, la productividad acadé- } \\
\text { mica y la orientación del programa. }\end{array}$ & $\begin{array}{l}\text { Institución con mayor número de programas reconoci- } \\
\text { dos por su calidad en el Estado de Sonora. } \\
\text { Categorías de compromiso institucional e infraestruc- } \\
\text { tura están en cumplimiento en los programas. }\end{array}$ \\
\hline Debilidades & Amenazas \\
\hline $\begin{array}{l}\text { El seguimiento de egresados es un criterio en vías } \\
\text { de cumplimiento en la mayor parte de los pro- } \\
\text { gramas. } \\
\text { La baja matrícula activa es un factor de relevan- } \\
\text { cia. } \\
\text { La baja vinculación con el sector productivo. }\end{array}$ & $\begin{array}{l}\text { El 2020, } 18 \text { de los programas tendrán que pasar por el } \\
\text { proceso de re-evaluación. }\end{array}$ \\
\hline
\end{tabular}

Fuente: Elaboración propia con base en datos de la Universidad de Sonora.

Tabla 4. Cumplimiento de criterios para cada categoría de evaluación del PNPC, según la división a la que pertenecen los posgrados de la Universidad de Sonora. 2015-2018

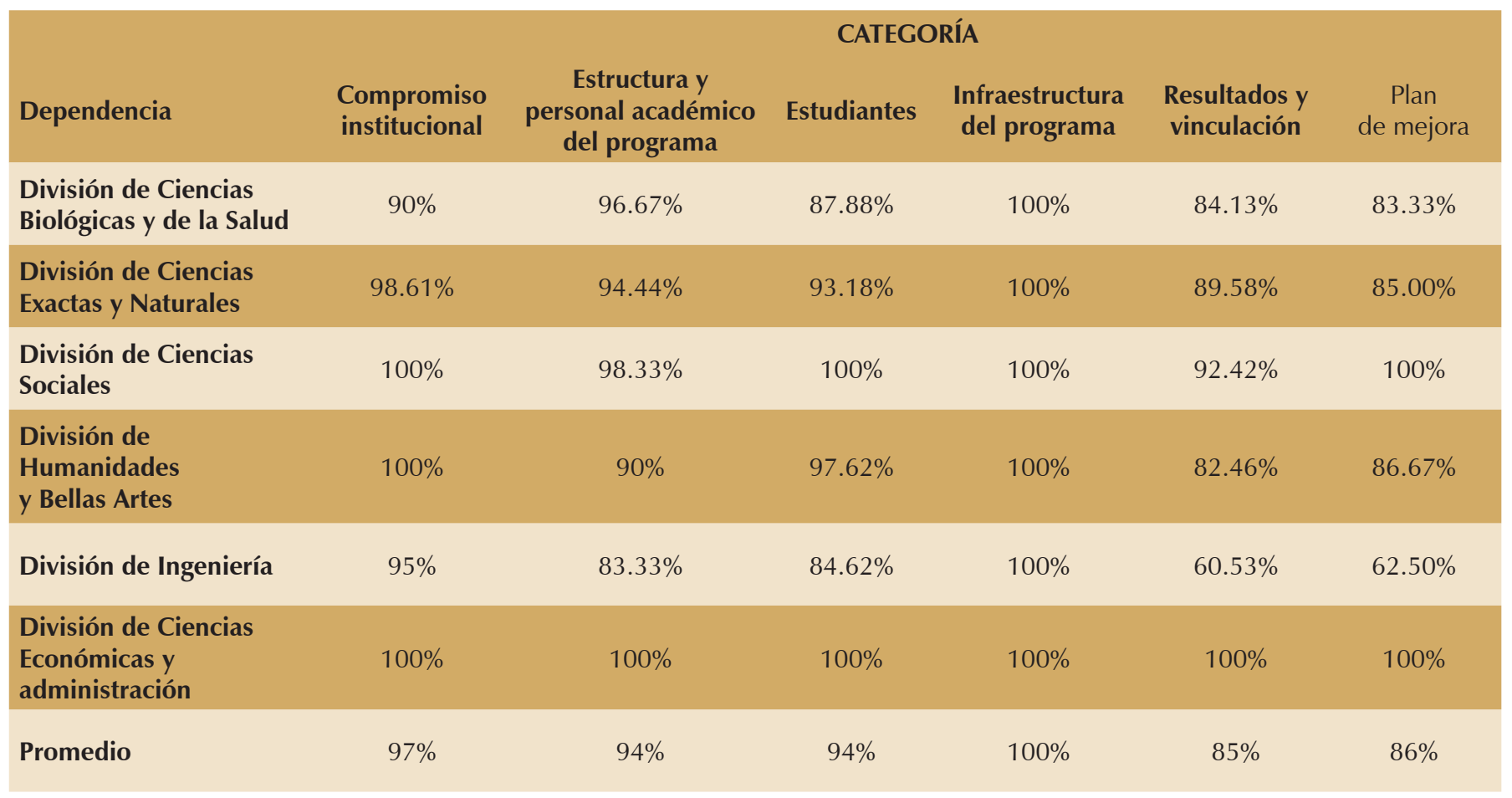

Fuente: Elaboración propia con base en los datos de los dictámenes de evaluación del PNPC de los programas de la Universidad de Sonora. 2015-2018 
No se encontraron amenazas que pudieran poner en juego la permanencia de los programas de posgrado en el PNPC, cabe mencionar que para el año 2020, 18 programas tendrán que re-evaluarse para mantener su nivel.

\section{CONCLUSIONES}

Hasta hace pocos años se empezaron a realizar estudios relacionados con este tema, un hecho que así lo constata, es que los dictámenes de evaluación de los programas no solo los de posgrado sino también la licenciatura, eran considerados documentos confidenciales dentro de las IES. Es por esto que se debe de reconocer la transparencia en la información de la Universidad de Sonora, al otorgar acceso abierto a la información.

Es necesario hacer énfasis en identificar las estrategias utilizadas por los programas con los niveles superiores del PNPC, de cómo estos fueron cumpliendo los criterios en los cuales tuvieron resultados negativos en las primeras evaluaciones, esto para buscar establecer acciones de mejora para los programas de la institución que aún tengan estos criterios en proceso de cumplimiento actualmente, o establecer las bases para los programas futuros que estén por iniciar la búsqueda de su reconocimiento dentro del PNPC.

\section{BIBLIOGRAFIA}

Barbón, O., Fernández J. (2017). Rol de la Gestión Educativa Estratégica en la Gestión del Conocimiento, la Ciencia, la Tecnología y la Innovación e la Educación Superior. Educación Médica. Volume 19, Issue 1, January-February 2018, Pages 51-55. DOI: https://doi.org/10.1016/j. edumed.2016.12.001

Barroso, F. (2011). Gestión del conocimiento en instituciones de educación superior y centros de investigación científica en el estado de Yucatán. Congreso internacional de contaduría, administración, e informática, D.F., México. Recuperado de http://congreso.investiga.fca.unam.mx/ docs/xvi/docs/9E.pdf

Careaga M., Meyer E., Badilla M., Jiménez L., y Sepúlveda E., (2017). Quality Assurance for Postgraduate Programs: Design of a Model Applied on a University in Chile. International Review of Reserch in Open and Distributed Learning. Volume 18, Number 1, February 2017. DOI: https://doi.org/10.19173/irrodl.v18i1.2670.

Colin O. L. (2002). Las normas ISO 9000:2000 de Sistemas de Gestión de la Calidad. Boletín IIE, julio-agosto, 182188. Recuperado de http://www.iie.org.mx/bollSO02/ tecni2.pdf

Consejo Nacional de Ciencia y Tecnología [Conacyt]. (2019). Programa Nacional de Posgrados de Calidad. Recuperado de https://www.conacyt.gob.mx/index.php/ becas-y-posgrados/programa-nacional-de-posgradosde-calidad.

Consejo Nacional de Ciencia y Tecnología [Conacyt]. (2020a). Marco de referencia para la evaluación y seguimiento de programas de nuevo ingreso modalidad escolarizada. Recuperado de https://www.conacyt.gob. $\mathrm{mx} /$ index.php/el-conacyt/convocatorias-y-resultadosconacyt/convocatorias-pnpc/marcos-de-referenciapnpc/19842-marco-referencia-evaluacion-programasnuevo-ingre/file

Consejo Nacional de Ciencia y Tecnología [Conacyt]. (2020b) Padrón del Programa Nacional de Posgrados de Calidad. Recuperado de http://svrtmp.main.conacyt.mx/ ConsultasPNPC/padron-pnpc.php

Fatemeh, H., Mohammad, R., Azam, A. (2011). The Quality Curriculum Evaluation in Postgraduate Studies of Educational Management and Planning in the Public Universities of Tehran City. Procedia - Social and Behavioral Sciences. Volume 15, 2011, Pages 3723-3730. DOI: https://doi.org/10.1016/j.sbspro.2011.04.363.

Fernández, N. (2007). Educación Superior y Calidad en América Latina y Argentina. Los procesos de evaluación y acreditación. ISBN: 978-987-1172-18-4 Buenos Aires: Eduntref.

Hanushek E. A. and Wößmann L. (2007). The Role of Education Quality in Economic Growth. World Bank Policy Research Working Paper 4122, February 2007. Recuperado de https://elibrary.worldbank.org/doi/ pdf/10.1596/1813-9450-4122

ISO (2015). Sistema de Gestión de Calidad - Fundamentos y vocabulario (NMX-CC-9000-IMNC-2015). Recuperado de http://serviciosweb.unevt.edu. $\mathrm{mx} / \mathrm{SGC} / \mathrm{SisGesCal} / 1 . \% 20 \mathrm{MANUAL} \% 20 \mathrm{DE} \% 20$ CALIDAD/1.2\%20COORDINACION\%20DE\%20 CALDIAD/Normas/NMX-CC-9000-IMNC-2015_E.pdf

OECD (2007). Insights Human Capital: how what you know shapes your life. ISBN-92-64-029095 (C) OECD 2007. Recuperado de https://www.oecd.org/ insights/38435951.pdf

OCDE (2011). Regions and Innovation Policy. Paris, OECD. Recuperado de http://www.oecd.org/innovation/ reviewsofregionalinnovationregionsandinnovationpolicy. htm

Pérez, A. (2007). La evaluación y la universidad como objeto de estudio. Avaliação, Campinas; Sorocaba, SP, v. 12, n. 4, p. 583-596, dez. 2007. 585-587. Recuperado de http://www.scielo.br/pdf/aval/v12n4/a03v12n4.pdf

PNPC (2011). Glosario de términos del PNPC para programas de posgrado escolarizados. SEP, CONACYT. Recuperado de http://dsia.uv.mx/sipo/Material_apoyo/ Glosario_Escolarizada.pdf

Secretaría de la Función Pública [SFP] (2018). Marco Conceptual sobre Gestión del Conocimiento. Recuperado de https://usp.funcionpublica.gob.mx/html/ Documentacion-DGDHSPC/AutogestionConocimiento/ MarcoConceptual_GestionConocimiento.pdf 
Taraneh, E., Yasaman, M., Reza, B., Abbas, R. (2012). Measuring Service Quality of Islamic Azad University of Mazandaran using SERVQUAL Model. Iranian Journal of Management Studies (IJMS) Vol.6, No.1, January 2013, pp: 99-116. Recuperado de https://ijms.ut.ac.ir/ rticle_30126_374900c5dc195493534884a7cb9eebc8. pdf

Tarí Guilló, J.J. y García Fernández, M. (2009). Dimensiones de la gestión del conocimiento y de la gestión de calidad: Una revisión de literatura. Investigaciones Europeas de Dirección y Economía de la Empresa. Vol. 15, No.3, 2009, pp. 135-148, ISSN:1135-2523.
United Nations Educational, Scientific and Cultural Organization (UNESCO). (2009). Conferencia mundial sobre educación superior 2009: "Las Nuevas Dinámicas de la Educación Superior y de la Investigación para el Cambio Social y el Desarrollo" [World conference on higher education 2009: "The New Dynamics of Higher Education and Research for Social Change and Development"]. París: Unesco. Recuperado de http:// www.unesco.org/education/wche/CMES_Folleto_ informativo_25032009.pdf

Universidad de Sonora [UNISON]. (2020). Dirección de Investigación y Posgrado (2020). Recuperado de http:// www.investigacion.uson.mx/?p=24 